\title{
Zukunft der Facharbeit
}

\author{
GERHARD BOSCH, THOMAS HAIPETER
}

$S$ eit mittlerweile zwei Jahrzehnten wird in der arbeitssoziologischen und bildungspädagogischen Diskussion das Ende der beruflichen Facharbeit in Deutschland heraufbeschworen. Demnach hat die Facharbeit als Fundament des deutschen Produktionsmodells ausgedient. Berufliche Qualifikationen taugten nicht mehr als Wettbewerbsvorteil in der internationalen Konkurrenz und als Grundlage der Organisation der Arbeit in den Betrieben. Die Begründungen für diese Diagnose sind vielfältig, konzentrieren sich aber allesamt auf Funktionsdefizite des deutschen Systems der beruflichen Ausbildung. Berufliche Facharbeit sei statisch und werde den laufend wechselnden Tätigkeitsanforderungen in flexiblen Beschäftigungssystemen nicht mehr gerecht; sie führe zu starrer Spezialisierung und blockiere eine prozessorientierte Reorganisation in den Unternehmen; und sie ignoriere die wachsende Bedeutung überfachlicher Kompetenzen, die in schulischer und tertiärer Bildung besser vermittelt würden als in der fachbezogenen Berufsausbildung.

Von solchen Endzeitvisionen der Facharbeit kann man sich getrost verabschieden. Facharbeit ist nach wie vor das qualifikatorische Rückgrat der Wettbewerbsfähigkeit der deutschen Industriebetriebe. Nicht von ungefähr richten deutsche Unternehmen bei der Gründung von Auslandsstandorten häufig in einem ersten Schritt Ausbildungsbetriebe ein, die den neu rekrutierten Beschäftigten fachliche Qualifikationen nach deutschen Ausbildungsstandards vermitteln sollen. Erst dann erfolgt der Aufbau der Betriebe und Produktionsanlagen. Diese Praxis ist Ausdruck der hohen Wertschätzung, die berufsfachliche Qualifikationen in den Unternehmen genießen. Und sie zeigt, dass sich das deutsche Ausbildungssystem in den letzten Jahrzehnten als überaus reformfähig erwiesen und viele Funktionsdefizite beseitigt hat: Erstens wurden neue fachliche und überfachliche Anforderungen in die Ausbildungsinhalte aufgenommen, um die Kooperations- und Prozesskompetenz wie auch die Interaktionskompetenz im Umgang mit Kunden zu verbessern. Zweitens wurden viele Berufe neu strukturiert und in gemeinsamen Kernqualifikationen gebündelt. Auf diese Weise wurden die fachliche Einsetzbarkeit der Beschäftigten und damit die funktionalen Flexibilitätsressourcen der Unternehmen deutlich erhöht. Und drittens schließlich wurden die Neuordnungsverfahren beschleunigt. Damit sind kontinuierliche Neuanpassungen der Ausbildungsordnungen an veränderte Bedarfe und Anforderungen möglich, die aus den Unternehmen signalisiert werden. Facharbeit ist übrigens nicht nur in der Industrie die unbestritten wichtigste Qualifikationsressource, sondern auch in den stetig wachsenden Dienstleistungsbranchen. Dies gilt für die traditionellen Branchen dieses Sektors wie den Einzelhandel oder das Bankgewerbe ohnehin, es trifft aber auch für die sozialen Dienstleistungen oder neue Branchen zu. Im IT-Sektor haben sich die vor gut einem Jahrzehnt geschaffenen neuen Berufe als Erfolgsmodell erwiesen, und analoge Entwicklungen finden sich in Fitnesscentern, Veranstaltungsbüros oder Werbeunternehmen.

Facharbeit ist kein überkommenes Relikt vergangener Tage. Dies ist die erste wichtige Botschaft, die mit diesem Schwerpunktheft vermittelt werden soll. Daran knüpft eine zweite Botschaft an: Die Zukunft der Facharbeit ist kein Selbstläufer. Vielmehr steht die berufliche Facharbeit trotz der aufgezeigten großen Reformleistungen vor fundamentalen $\mathrm{He}$ rausforderungen. Ihre Zukunft wird deshalb entscheidend davon abhängen, welche Interessen und Strategien die für das Qualifikationssystem relevanten Akteure - Sozialpartner, Unternehmen, Staat, aber auch Jugendliche und deren Familien mit ihren Bildungs- und Ausbildungsentscheidungen - verfolgen. Drei Herausforderungen, die in den Beiträgen dieses Heftes vertieft werden, sind evident. Die Erste ist die bröckelnde Stärke der Sozialpartner. Für sie wird es immer schwieriger, die kontinuierliche Reform von Ausbildungsordnungen zu gestalten. Und sie können zunehmend seltener tarifliche Löhne und Arbeitsstandards garantieren, die auch ein faires Entgelt für fachlich gute Arbeit versprechen. Zweitens werden von der Politik höhere Akademikerquoten angestrebt. Leitbilder dafür sind Länder, denen es an einer Tradition der beruflichen Facharbeit fehlt und die die fachliche Qualifizierung ganz in die tertiäre Ausbildung verlagert haben. Durch die Bologna-Reformen an den Universitäten und die Einführung des Bachelor ist der dualen Berufsausbildung zudem eine neue Konkurrenz erwachsen. Jugendliche entscheiden sich vermehrt für ein Studium. Der beruflichen Ausbildung droht deshalb ein Rekrutierungsproblem. Duale Studiengänge, in denen berufliche und akademische Bildung kombiniert werden, sind eine Antwort der Unternehmen, Facharbeit trotz dieses Wandels der Bildungspräferenzen zu erhalten. Die dritte Herausforderung schließlich ist die Entstehung eines europäischen Arbeitsmarktes und die Vergleichbarkeit der nationalen Qualifikationsstandards durch einen Europäischen Qualifikationsrahmen. In den meisten Mitgliedstaaten wird akademische Ausbildung strukturell höher gewichtet als die berufsfachliche. Umso bedeutender ist die Gleichstellung der Aufstiegsfortbildungen mit dem Bachelor im Deutschen Qualifikationsrahmen. Facharbeit hat also eine Zukunft, sie ist aber politisch hochgradig umstritten und sie muss aktiv verteidigt und kontinuierlich neu gestaltet werden.

\section{KONZEPT UND KOORDINATION DES SCHWERPUNKTHEFTES}

GERHARD BOSCH, Prof. Dr., ist Geschäftsführender Direktor des Institut Arbeit und Qualifikation (IAQ) an der Universität Duisburg-Essen.

gerhard.bosch@uni-due.de

THOMAS HAIPETER, PD Dr., leitet die Forschungsabteilung Arbeitszeit und Arbeitsorganisation am IAQ.

thomas.haipeter@uni-due.de 